\title{
Liquid-Liquid Diffusion-Assisted Crystallization: A Fast and Versatile Approach Toward High Quality Mixed Quantum Dot-Salt Crystals
}

\author{
Marcus Adam, Zhiyu Wang, Aliaksei Dubavik, Gordon M. Stachowski, Christian Meerbach, \\ Zeliha Soran-Erdem, Christin Rengers, Hilmi Volkan Demir, Nikolai Gaponik, * \\ and Alexander Eychmüller
}

Here, a new, fast, and versatile method for the incorporation of colloidal quantum dots (QDs) into ionic matrices enabled by liquid-liquid diffusion is demonstrated. QDs bear a huge potential for numerous applications thanks to their unique chemical and physical properties. However, stability and processability are essential for their successful use in these applications. Incorporating QDs into a tight and chemically robust ionic matrix is one possible approach to increase both their stability and processability. With the proposed liquid-liquid diffusion-assisted crystallization (LLDC), substantially accelerated ionic crystallization of the QDs is shown, reducing the crystallization time needed by one order of magnitude. This fast process allows to incorporate even the less stable colloids including initially oil-based ligandexchanged QDs into salt matrices. Furthermore, in a modified two-step approach, the seed-mediated LLDC provides the ability to incorporate oilbased QDs directly into ionic matrices without a prior phase transfer. Finally, making use of their processability, a proof-of-concept white light emitting diode with LLDC-based mixed QD-salt films as an excellent color-conversion layer is demonstrated. These findings suggest that the LLDC offers a robust, adaptable, and rapid technique for obtaining high quality QD-salts.

approaches, ${ }^{[15,16]}$ efficiency, ${ }^{[17,18]}$ extending their spectral range ${ }^{[19-21]}$ and environmental friendliness using less toxic materials $^{[22-24]}$ sparked industrial applications. Those started with the demonstration of a 40 in. display prototype presented by Samsung ${ }^{[25]}$ and followed by the foundation of QD Vision, Inc., ${ }^{[26]}$ whose QDs are now used in the latest series of Sony products. All of the mentioned applications require long-term stability of the QDs under various conditions including high temperatures as well as high intensity illumination. Packaging of the QDs within polymer or inorganic matrices is one way to address these issues, while improving the processability of the QDs at the same time. Commonly used polymers, such as polystyrene and poly(methylmethacrylate) $)^{[27]}$ are relatively less stable and tight in comparison to their inorganic counterparts. Inorganic matrices on the other hand can incorporate the QDs directly from their melt, e.g., using the

\section{Introduction}

In the last three decades since their first reports appeared, ${ }^{[1-5]}$ colloidal semiconductor quantum dots (QDs) attracted continuously increasing interest in different fields, including photonics $^{[6-10]}$ and optoelectronics. ${ }^{[11-14]}$ Improving the characteristics and quality of QDs regarding synthetic
Czochralski approach, ${ }^{[28]}$ coated as a thin film directly on their surface ${ }^{[29,30]}$ or, as recently developed by our group, grown as mixed crystals at ambient temperatures from a saturated salt solution. ${ }^{[31]}$ Using this method, different types of QDs can be incorporated due to the low thermal stress and, by choosing the proper matrix-QD system, the photoluminescence quantum yields (PL-QYs) are enhanced upon incorporation. ${ }^{[32,33]}$ The
M. Adam, Dr. A. Dubavik, G. M. Stachowski, C. Meerbach, C. Rengers, Prof. N. Gaponik, Prof. A. Eychmüller

Physical Chemistry

TU Dresden, Bergstr. 66b, 01062 Dresden, Germany

E-mail: nikolai.gaponik@chemie.tu-dresden.de

Prof. Z. Wang

Carbon Research Laboratory

Liaoning Key Lab for Energy Materials and Chemical Engineering

State Key Lab of Fine Chemicals

Dalian University of Technology

Dalian 116024, P.R. China

DOI: 10.1002/adfm.20150055
Z. Soran-Erdem, Prof. H. V. Demir

Department of Physics, Department of Electrical and Electronics Engineering and UNAM-Institute of Materials Science and Nanotechnology Bilkent University TR-06800 Ankara, Turkey

Prof. H. V. Demir

LUMINOUS! Center of Excellence for Semiconductor Lighting and Displays School of Electrical and Electronic Engineering and School of Physical and Mathematical Sciences Nanyang Technological University Singapore 639798, Singapore 
weak aspect of this approach is the time consuming crystallization of typically 1-4 weeks, which also requires extremely high stability of the QDs within the saturated salt solution during this long period of crystallization. This consequently limits the set of the QDs that can be successfully incorporated into ionic matrices.

In this paper, we report a versatile and fast method of producing high-quality QD-salt mixed crystals, relying on the diffusion and solubility change of inorganic salts (e.g., $\mathrm{NaCl}$ ) in solvents with various polarities. Due to the reduced solubility of $\mathrm{NaCl}$ in water by the interdiffusion of the worse solvent, the complete crystallization finishes within less than one day. With this, the time needed to produce mixed crystals was reduced by more than one order of magnitude in comparison to the times reported in refs. [31] and [33]. Reducing the crystallization time enables the use of even less stable QD colloids (e.g., ligand exchanged initially oil-based QDs). Moreover, to the best knowledge of the authors, for the first time, adapting a twostep seed-mediated liquid-liquid diffusion-assisted crystallization (LLDC), the direct application of oil-based QDs for crystallization with salts without a prior phase transfer into water was achieved. The resulting mixed crystals exhibit a stable and chemically tight matrix and can be produced using a variety of semiconductor colloids, including Cd-free QDs, which may greatly extend the application of the QDs in photonic applications.

\section{Results and Discussions}

As mentioned above, our newly established LLDC-method for the preparation of QD-salt mixed crystals takes roughly one day to be completed, which is a distinct reduction compared to former, standard crystallization approaches. ${ }^{[31,33-35]}$ The basic principle behind this method is the difference in solubility of $\mathrm{NaCl}$ in water and organic solvents with lower polarity, as shown in Table 1. Methanol is chosen as a solvent to demonstrate our concept due to its miscibility with water while having a much lower solubility of the $\mathrm{NaCl}$ in comparison to water. Furthermore, the QDs are stable within $\mathrm{MeOH}$ long enough to allow the crystallization to complete.

\subsection{Preparation of Mixed Crystals Using Aqueous-Based CdTe QDs}

CdTe QDs were first employed for the preparation of mixed crystals by the LLDC-approach due to their relatively high stability in saturated salt solutions. As schematically shown in

Table 1. Solubility of $\mathrm{NaCl}$ in different solvents. ${ }^{[36]}$

\begin{tabular}{lc}
\hline Solvent & $\begin{array}{c}\text { Soluble } \mathrm{NaCl} \\
{\left[\mathrm{g} \mathrm{kg}^{-1} \text { solvent }\right]}\end{array}$ \\
\hline Water & 360 \\
Glycerin & 83 \\
Methanol & 14 \\
Ethanol & 0.65 \\
\hline
\end{tabular}
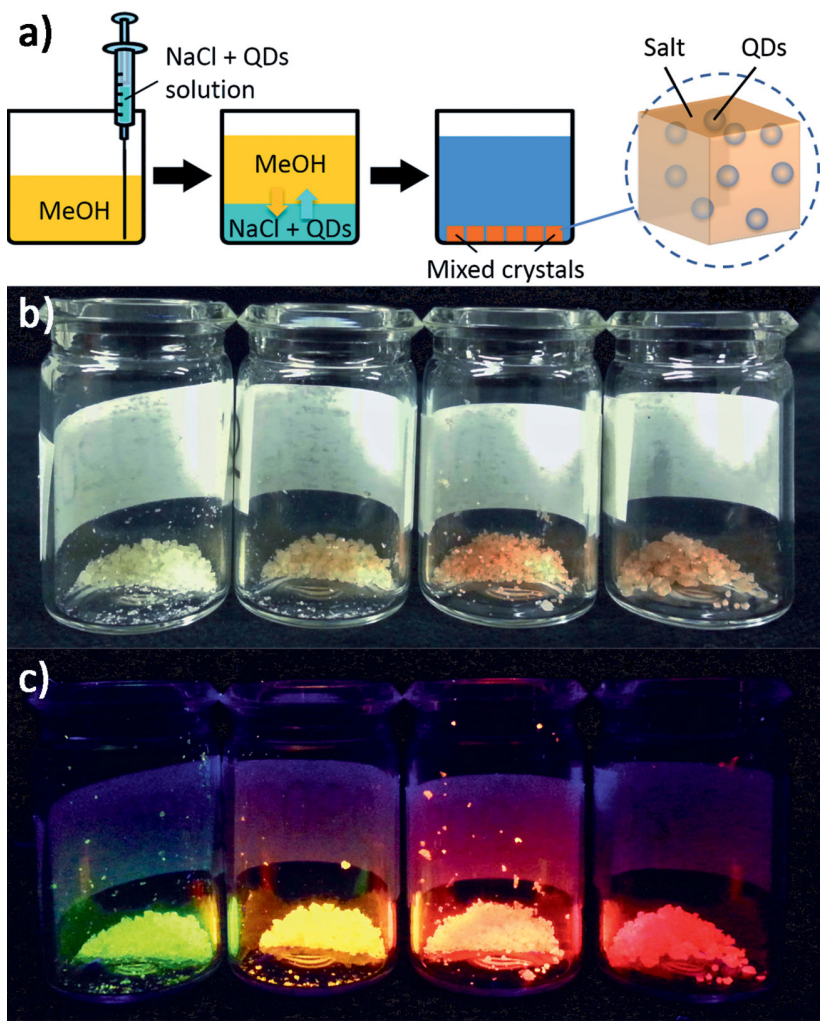

Figure 1. a) Schematic drawing of the LLDC-approach using the diffusion of $\mathrm{MeOH}$ through a stable interface layer into an aqueous solution containing $\mathrm{NaCl}$ and $\mathrm{CdTe}$ QDs. True color images of an exemplarily chosen set of $\mathrm{NaCl}$-based mixed crystals containing differently sized CdTe QDs under b) daylight and c) $365 \mathrm{~nm}$ UV-illumination.

Figure 1a, a stable interface between the $\mathrm{MeOH}$ solution and the underlying NaCl-QD mixture instead of an instantaneous mixing is crucial for a successful crystallization. Through the subsequent diffusion of $\mathrm{MeOH}$ into water and the thereby reduced solubility, $\mathrm{NaCl}$ crystals with incorporated CdTe QDs are formed within hours. Four exemplarily chosen QD-salt mixed crystal samples are shown in Figure 1b,c, proving their color and intense emission under daylight and UV illumination, respectively.

Figure 2 enables a magnified view of the mixed crystals. Column (a) represents top-view true color images under UVexcitation at $365 \mathrm{~nm}$, while column (b) shows fluorescence microscopic images of the green-, yellow-, orange-, and redemitting mixed crystals. These microscopic images clearly reveal that the QDs are evenly distributed within the mixed crystals as well as an octahedral crystal shape of the host material. In general, $\mathrm{NaCl}$ forms cubic crystals, while the addition of small, coordinating molecules like the stabilizing agent mercaptopropionic acid (MPA) used for the synthesis of the CdTe QDs causes a change in shape toward octahedrons. ${ }^{[37]}$ The formed mixed crystals are small, in a size regime of the edges of about $0.5 \mathrm{~mm}$, e.g., in Figure 2, column (b), which is a size reduction in comparison to the standard crystallization approach we presented earlier where mixed crystals with sizes larger than $1 \mathrm{~cm}$ are obtained. ${ }^{[31]}$ Such smaller crystallite sizes can be explained 


\section{Column a) Column b)}

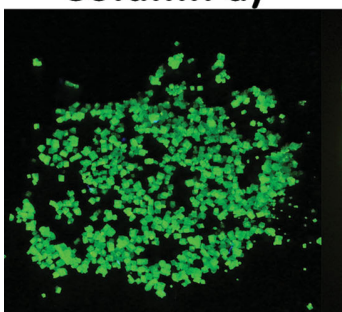

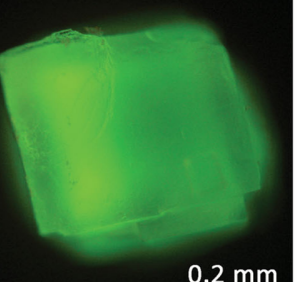

$0.2 \mathrm{~mm}$
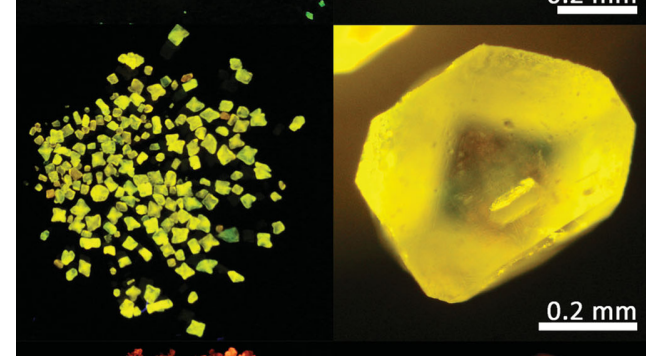

$0.2 \mathrm{~mm}$
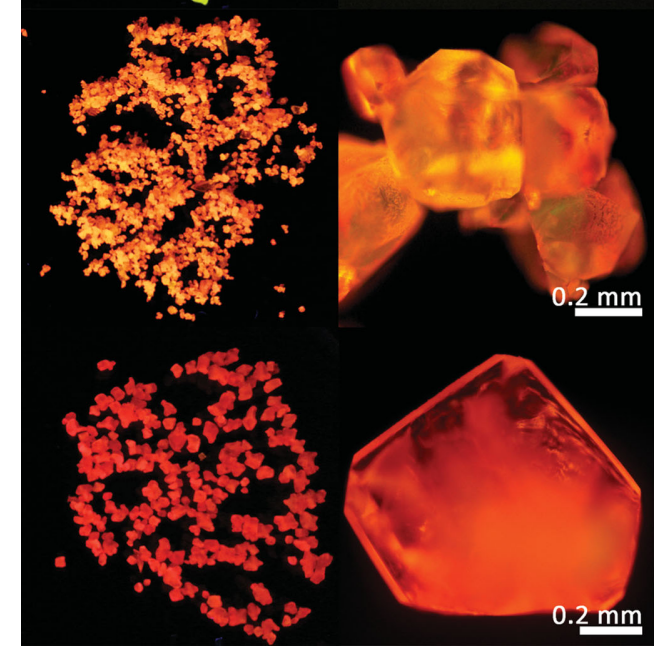

Figure 2. True color macroscopic images (column a)) and fluorescence microscopy images (column b)) of mixed crystals emitting in different colors under $365 \mathrm{~nm}$ UV-excitation. Column c) displays the PL-spectra of the initial CdTe QDs (dashed black lines) as well as the corresponding mixed crystals (red lines).

by the fast crystallization rate in the $\mathrm{MeOH}$-diffusion induced oversaturation of the $\mathrm{NaCl}$ solution, which leads to the simultaneous formation of a larger amount of crystal seeds. Furthermore, the overall reduced amount of $\mathrm{NaCl}$ within the system also favors the formation of smaller mixed crystals. Column (c) of Figure 2 shows the corresponding PL-spectra of the mixed crystals (red lines) in comparison to the PL-spectra of the pure QDs in solution (dashed black lines). All four samples show a minimal red shift in their emission maxima upon incorporation into the salt matrix, which is associated with the change of the dielectric constant of the surrounding media. ${ }^{[31]}$ The PL QY of the CdTe QDs did not change in the course of transferring them from the parent solution to the $\mathrm{NaCl}$ crystals. This suggests that the fast crystallization procedure does not allow to form a complete passivating $\mathrm{CdCl}_{x}$-shell since this would result in an increase of the PL QY as observed in our recent study. ${ }^{[32]}$ Using LLDC, the production of mixed crystals can also be performed using other solvents, e.g., ethanol and other salts, e.g., $\mathrm{KCl}$. In both cases, the resulting mixed crystals are much more powder-like, as can be seen from Figures S2 and S3, Supporting Information, respectively. This can possibly be explained with the lower solubility of $\mathrm{NaCl}$ in EtOH and $\mathrm{KCl}$ in $\mathrm{MeOH}$ in comparison to $\mathrm{NaCl}$ in $\mathrm{MeOH}$, yielding an even larger number of crystallization seeds during the diffusion of the alcohols into the underlying water phase and therefore leading to the formation of more but smaller mixed crystals.

Transmission electron microscopy (TEM) imaging of QD-salt mixed crystals is difficult because the $\mathrm{NaCl}$ matrix tends to get charged under the focused electron beam, which causes fast melting of the sample. To solve this problem, a composite of resin and mixed crystals was prepared for TEM measurements. The composite was cut into sheets as thin as $200 \mathrm{~nm}$ to enable the transmission of the electron beam, while the presence of the surrounding resin may protect the mixed crystals from melting for at least several minutes. A TEM image of the mixed crystal within such a thin sheet is shown in Figure 3. The TEM confirms the successful incorporation and a relatively even and nonaggregated distribution of the QDs within the $\mathrm{NaCl}$ matrix, although the LLDC-process is fast. The observation of the separated integration of the QDs into the salt matrix is in agreement with the optical studies showing only a slight shift in the PL spectra going from the dispersion to the solid salt.

\subsection{Preparation of Mixed Crystals Using Oil-Based QDs After Ligand Exchange}

In recent years, besides aqueous synthetic routes, hot injection techniques have been widely investigated to obtain high-quality QDs with small size dispersion and high PL-QYs (>70\%). These QDs are mainly stabilized in nonpolar solvents by long-chain alkyl phosphines (e.g., trioctylphosphine (TOP)/TOPO) and alkanoic acids. Exchanging the ligands on the surface with shorter, polar molecules like MPA is one efficient way to make these QDs water-soluble and accessible for co-crystallization with salt. However, this approach has a limitation as the stability of ligand-exchanged QDs in salt solutions is moderate and thus not always compatible with the conventionally used long time crystallization approach. ${ }^{[32]}$ At the same time, using the LLDC, this inherent drawback of the ligand exchanged QDs can be overcome as a result of the acceleration of the crystallization procedure by more than an order of magnitude. Figure 4 shows the results for $\mathrm{NaCl}$ mixed crystals containing ligand-exchanged MPA-capped CdSe/ZnS core/shell QDs with an alloyed gradient shell $(\mathrm{a}-\mathrm{c})$ as well as InZnP/GaP/ ZnS core/shell/shell QDs (d-f). At first sight, a more cubic-like structure of the mixed crystals is obtained (Figure 4b,e), indicating a much lower amount of free MPA within the parental 


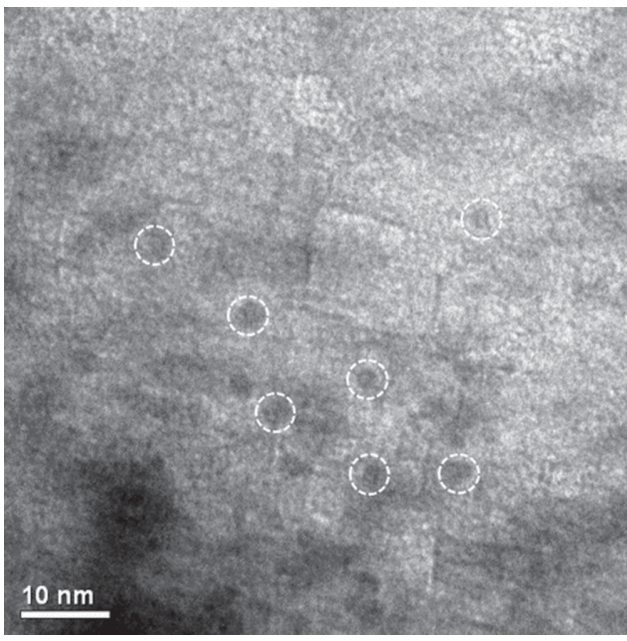

Figure 3. TEM image of $\mathrm{CdTe} \mathrm{QDs}$ in a $\mathrm{NaCl}$ crystal (sample shown in Figure 7c) using ultramicrotomy to prepare thin slides embedded in a protective resin sheet. Some of the QDs are highlighted with a circle as a guide for the eye.

solution. A smaller amount of ligands on the surface is one major parameter causing the reduced stability of the QDs within the salt solutions. Using the LLDC-method, these materials can be made accessible for co-crystallization with $\mathrm{NaCl}$ without larger losses due to the shorter time needed to complete the crystallization process. The trade-off which has to be made using ligand exchanged QDs for the incorporation into solid matrices is the inherent reduction of the PL-QY during the ligand exchange. During the exchange step, the PL-QY is reduced to $25 \%-50 \%$ of its initial value.

\subsection{Preparation of Mixed Crystals Using Oil-Based QDs Without Prior Ligand Exchange}

To overcome the critical step of exchanging ligands, we extended the LLDC-method to a two-step seed-mediated approach, as schematically illustrated in Figure 5. First, the addition of NaClsaturated $\mathrm{MeOH}$-solution into the diluted QD-solution causes a drastically reduced solubility of $\mathrm{NaCl}$, creating $\mathrm{NaCl}$ seed crystals as shown as turbidity in Figure S1, Supporting Information. During their formation, most of the QDs are adsorbed on the surface of the $\mathrm{NaCl}$ seeds to reduce the surface-free energy. These small crystallites are already usable for further applications, but due to the large amount of QDs solely on the surface, these seeds do not provide a thorough protection of the QDs. However, they are ideal crystal seeds for the subsequent growth of mixed crystals by simply repeating the LLDC-approach. We then perform the LLDC as a second step by dispersing the seeds within pure $\mathrm{MeOH}$ and injecting an underlying layer of $\mathrm{NaCl}$-solution. $\mathrm{MeOH}$ is used as the mediation solvent due to its miscibility with water and nonpolar organic solvents like $\mathrm{CHCl}_{3}$ while it is a nonsolvent for the QDs themselves.

Figure 6 shows mixed $\mathrm{NaCl}$-based crystals composed of either green-, yellow-, or red-emitting oil-based CdSe/ZnS QDs without ligand exchange prepared via the seed-mediated LLDC-method. As can be seen from the microscopic images (Figure 6a,d,g), the mixed crystals exhibit a pure color emission and a cubic shape. This shape is expected, since no small coordinating molecules, which would alter the shape toward octahedrons, are present during the complete crystallization process. Second, and in comparison to the discussed LLDC results above, the emission centers are not evenly distributed within the mixed crystals, but mostly concentrated around the initial seed, confirming our conception of the QDs adsorption onto the seeds in the first step and their subsequent coverage with mainly pure $\mathrm{NaCl}$ during the second-step LLDC, as shown in Figure $6 \mathrm{~b}, \mathrm{e}, \mathrm{h}$. The corresponding PL-spectra are shown in Figure $6 c$,f,i. These spectra exhibit a slight red shift, explained by the change of the surrounding media as discussed above. No signs of aggregation like broadening, intense red shifts, or scattering effects are observed. Figure $6 j$ shows a true color image of the mixed crystals under $365 \mathrm{~nm}$ excitation, proving their intense emission. For a further understanding of how the optical quality of the used QDs develops upon the incorporation using the LLDC-method, the changes in the PL-lifetime (PL-LT) as well as the PL-QY were investigated. Red- and green-emitting samples shown in Figure 6 were chosen as representative examples. Indeed, according to ref. [38], the used one-pot synthesis yields QDs with different emission colors by adjusting the ratios of the precursors while the overall size of the QDs stays nearly constant. Therefore, adjusting the precursor ratios only changes the ratio of core-size to shell-thickness, meaning that yellow-emitting QDs will behave like an intermediate of red- and green-emitting QDs. The measurements showed (Figure S4, Supporting Information) that the PL-LT dropped from $27.65 \mathrm{~ns}$ in $\mathrm{CHCl}_{3}$ to $20.04 \mathrm{~ns}$ in $\mathrm{NaCl}$ and from 23.34 to $7.45 \mathrm{~ns}$ for the green and the red QDs, respectively. At the same time, the decrease in the PL-QY was relatively
Figure 4. a,d) True color images and b,e) microscopic images under $365 \mathrm{~nm}$ UV-excitation as well as c,f) PL-spectra of mixed crystals with a-c) CdSe/ZnS QDs or d-f) InZnP/GaP/ZnS QDs. The mixed crystals are prepared after replacing the long-chain ligands with MPA. 


\section{Seed-ss]edjajed LLDC}

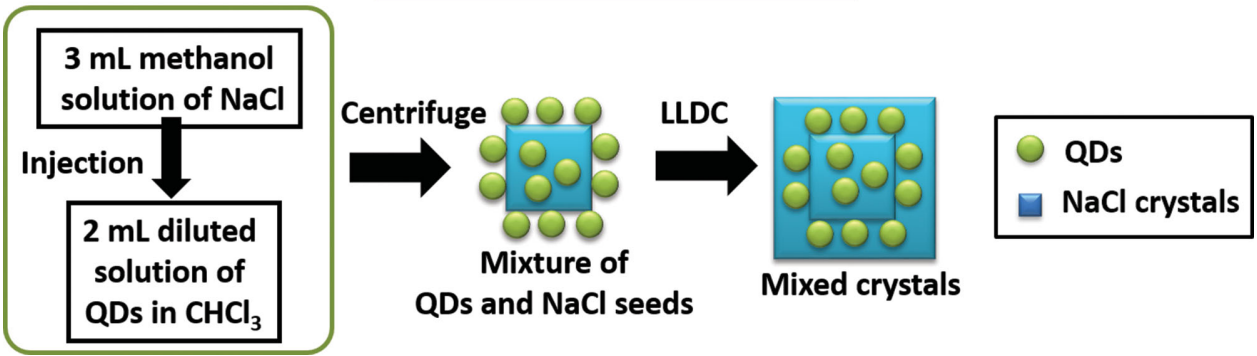

Figure 5. Schematic illustration of the seed-mediated LLDC-method which enables the direct usage of oil-based QDs for crystallization without a prior ligand exchange. In the first step, QDs are adsorbed at the surface of the formed $\mathrm{NaCl}$ seeds, while the LLDC in the second step provides a complete encapsulation of the QDs by growing the seeds to larger mixed crystals.

lower in both cases, i.e., from $42.2 \%$ in $\mathrm{CHCl}_{3}$ to $34.7 \%$ in $\mathrm{NaCl}$ and from $36.5 \%$ to $16.2 \%$ for the green and the red samples, respectively. This observation is in good agreement with the conception mentioned above in that the QDs are mainly

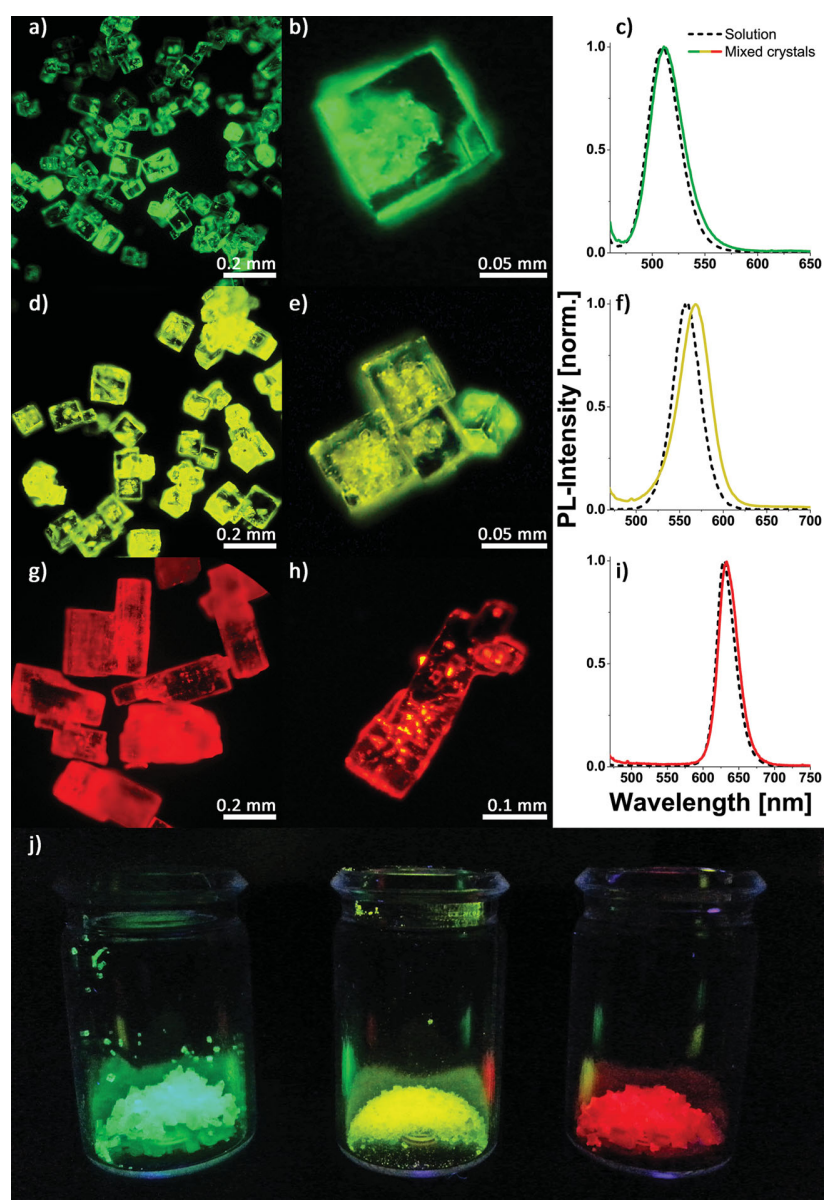

Figure 6. a,d,g) Microscopic images under $365 \mathrm{~nm}$ UV-excitation and $c, f, i)$ PL-spectra of green, yellow, and red emitting CdSe/ZnS QDs incorporated into $\mathrm{NaCl}$ without any prior ligand exchange using the seedmediated LLDC-approach. b,e,h) The presence of crystal seeds within the mixed crystals is visualized. Photograph j) shows a true color image of the corresponding mixed crystals under $365 \mathrm{~nm}$ UV excitation. concentrated around the initial crystallization seed formed in the first step. A close proximity between the individual QDs in such a shell results in a higher risk of self-quenching. In this respect, a stronger decrease in PL-QY for the red-emitting QDs can be explained by the thinner protecting ZnS-shell on their surfaces, making it easier for charge carriers to reach the surface for any kind of environment-related quenching process. On the other side, the used green-emitting QDs possess a thicker ZnS shell, confining the charge carriers more strongly within the core and reducing the overall possibility for environment-related quenching processes. ${ }^{[39]}$ To verify this hypothesis, we prepared mixed crystals using the two-step LLDC approach with bare, nonshelled CdSe QDs as emitting centers. As expected, these samples showed a complete loss of $\mathrm{PL}$ upon incorporation into $\mathrm{NaCl}$ crystals.

\subsection{Mixed Crystal Stability Test}

For these mixed QD-salts to be used as luminophores, a high level of stability is crucial. While it was previously shown that the incorporation of QDs into mixed crystals remarkably increases their photometric stability under illumination, ${ }^{[31]}$ the mixed crystals presented in this work need to prove that, even with the fast crystallization as in the LLDC, the QDs are fully incorporated into the $\mathrm{NaCl}$ matrix and that the resulting matrix is free of fractions. For the stability tests, mixed crystals as well as some CdSe/ZnS QDs in toluene were exposed to solutions of benzoyl peroxide in toluene. In the presence of the strong oxidant, the emission of the CdSe/ZnS QDs was fully quenched after $24 \mathrm{~h}$ (Figure 7a,b). The mixed crystals made of $\mathrm{NaCl}$ and either aqueous CdTe or oil-based CdSe/ $\mathrm{ZnS}$ QDs without prior ligand exchange were robust enough to withstand the oxidation in benzoyl peroxide solution under identical conditions (Figure 7c,d). Their emission intensity was almost unaltered during the oxidation test, showing a stability comparable to the mixed crystals made by the standard crystallization, as can be seen in Figure S5, Supporting Information.

Additionally, it is noted that the mixed crystals display a superior environmental stability. Their PL-QY was reduced by only a few percent of the initial intensity after storage under ambient conditions for more than one year. 


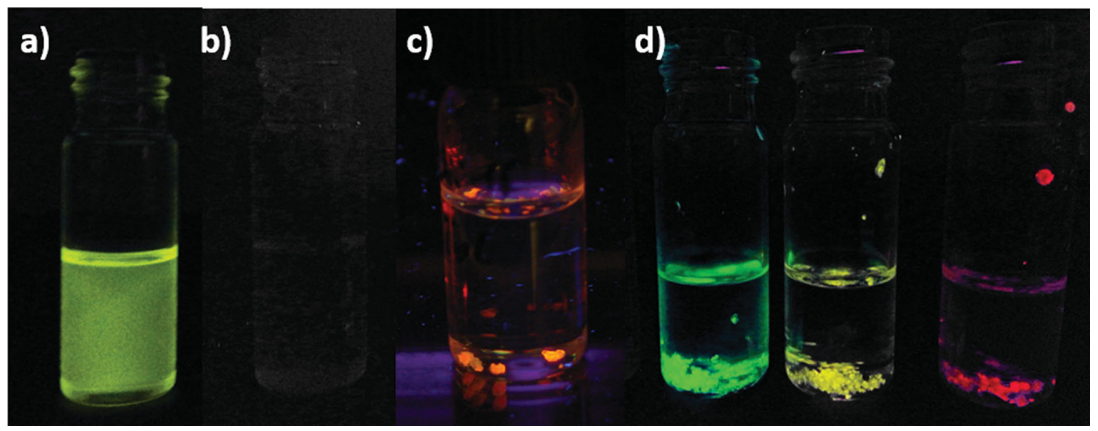

Figure 7. True color images of the stability test of the LLDC-produced mixed crystals using benzoyl peroxide as oxidizing agent under $365 \mathrm{~nm}$ UV excitation. A solution of CdSe/ZnS QDs in toluene a) before and b) after adding benzoyl peroxide for $24 \mathrm{~h}$; mixed crystals containing c) CdTe QDs and various d) CdSe/ZnS QD after adding benzoyl peroxide in toluene for $24 \mathrm{~h}$.

\subsection{Demonstration of a Color-Conversion White Light Emitting Diode}

Films of mixed QD-salts prepared by the seed-mediated LLDC method were integrated on a commercially available $1 \mathrm{~W} 460 \mathrm{~nm}$ blue-emitting InGaN LED in order to construct a proof-ofconcept color-conversion white LED. The emission spectrum of an exemplarily integrated device when electrically driven is presented in Figure 8a. Figure 8b displays the white LED under ambient illumination as well as during operation. Figure $8 \mathrm{c}, \mathrm{d}$ shows the LLDC-based mixed crystals used for the white LED preparation. Their durability and photostability is comparable to that of LEDs made with conventionally grown mixed crystals as reported by us ${ }^{[31]}$ and others. ${ }^{[33]}$ Moreover, no deterioration of the emission properties is observed after applying an on/off test for at least 20000 cycles. We note that further optimization regarding the color temperature and color rendering is possible, which will be the focus of our future work.

\section{Conclusion}

In summary, we demonstrate a versatile approach toward the fast preparation of highquality colloidal QD-salt mixed crystals. With the liquid-liquid diffusion-assisted process, both aqueous and oil-based QDs are accessible for the co-crystallization, extending the range of QDs that can be used while reducing the time needed for the preparation of the mixed crystals. We presented for the first time the incorporation into an ionic matrix of Cd-free semiconductor-based QDs (InZnP/GaP/ZnS) emitting in the visible range. Furthermore, by extending the LLDC-method into a two-step procedure, we achieved to directly incorporate oil-based QDs without a prior ligand exchange, overcoming the difficulties associated with the moderate stability and the lowering of the PL-QY of ligand-exchanged QDs. We verified that the QDs are incorporated into the mixed crystals and not just adsorbed on their surface and that the fast crystallization procedure does not affect the quality of the protective ionic matrix. Using the LLDC-based mixed crystals a proof-of-concept white LED has been made, showing the applicability of the mixed crystals as excellent color-conversion materials. Enabled by the LLDC, mixed crystals may have made another crucial step on their way to further and industrial exploitation.

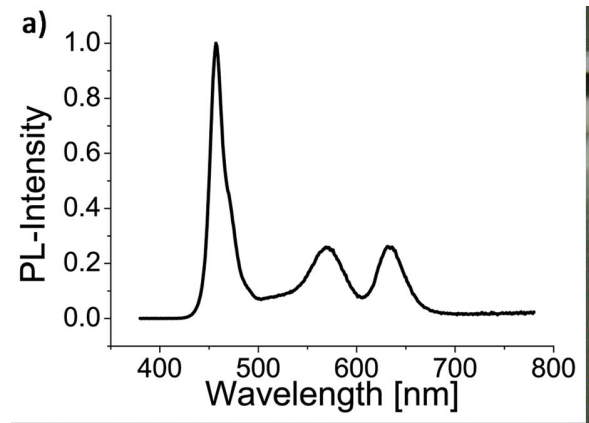

c)

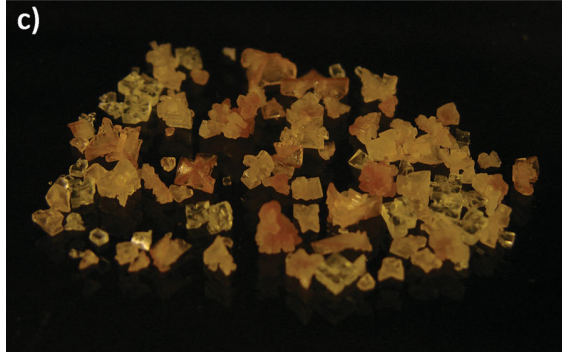

d)

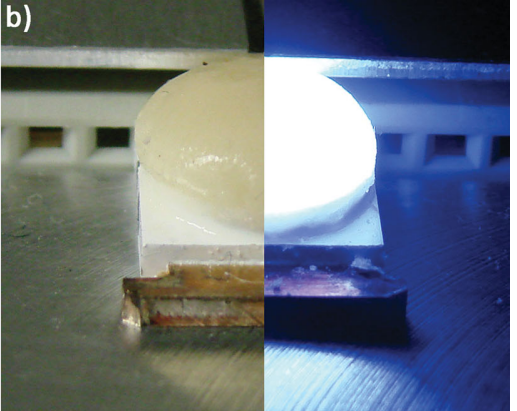

Figure 8. a) Emission spectrum of a proof-of-concept white LED using the seed-mediated LLDC-based QD-salt mixed crystals as color converters. b) Photograph shows a true color image of the resulting LED under ambient illumination (left part) and with the LED switched on (right part). Images of the mixed crystals under the illumination of a c) standard fluorescent lamp and d) $365 \mathrm{~nm}$ UV excitation are given for comparison.

\section{Experimental Section}

Chemicals and Apparatus: All chemicals used were of analytical grade or of the highest purity available. All aqueous solutions were prepared from Milli-Q water (Millipore). The $\mathrm{Al}_{2} \mathrm{Te}_{3}$ lumps employed for the generation of $\mathrm{H}_{2} \mathrm{Te}$ were purchased from CERAC Inc. As for the QDs, we

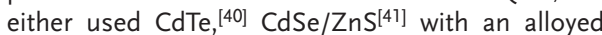
gradient shell, or core/shell/shell (CSS) InZnP/ $\mathrm{GaP} / \mathrm{ZnS}^{[42]}$ QDs, which were prepared according to previous publications.

Synthesis of QDs: Briefly, CdTe QDs were synthesized by dissolving $2.305 \mathrm{~g}(5.5 \mathrm{mmol})$ of $\mathrm{Cd}\left(\mathrm{ClO}_{4}\right)_{2} \cdot 6 \mathrm{H}_{2} \mathrm{O}$ in $250 \mathrm{~mL}$ water, adding $0.758 \mathrm{~g}$ (7.15 mmol) of mercaptopropionic acid (MPA) as the thiol stabilizer, followed by adjusting the $\mathrm{pH}$ to 12 using a $1 \mathrm{M}$ solution of $\mathrm{NaOH}$. The resulting solution was placed in a three-necked flask and deaerated with Ar for 30 min. The gaseous Te-precursor $\mathrm{H}_{2} \mathrm{Te}$ was generated by reacting $0.4 \mathrm{~g}$ (0.916 mmol) $\mathrm{Al}_{2} \mathrm{Te}_{3}$ with $10 \mathrm{~mL}$ of a $0.5 \mathrm{M} \mathrm{H}_{2} \mathrm{SO}_{4}$ solution and passed through the solution with a slow argon flow under vigorous stirring. The growth of the $\mathrm{CdTe}$ QDs proceeded under open air conditions at $100^{\circ} \mathrm{C}$ with a condenser attached. The obtained QD solutions were used as prepared with no additional cleaning or concentration steps and possessed PL-QYs of $40 \%-50 \%$. Green-emitting 
$\mathrm{CdSe} / \mathrm{ZnS} \mathrm{QDs}$ with an alloyed gradient shell were synthesized using $51.5 \mathrm{mg}(0.4 \mathrm{mmol}) \mathrm{CdO}, 733.6 \mathrm{mg}(4 \mathrm{mmol}) \mathrm{Zn}(\mathrm{OAc})_{2}, 5.5 \mathrm{~mL}$ of oleic acid (OA), and $20 \mathrm{~mL}$ of octadecene (ODE) mixed in a $50 \mathrm{~mL}$ three-necked flask. The suspension was degassed at $100{ }^{\circ} \mathrm{C}$ for $60 \mathrm{~min}$, backfilled with $\mathrm{Ar}$, and heated to $310^{\circ} \mathrm{C}$. Subsequently, $7.9 \mathrm{mg}$ $(0.1 \mathrm{mmol}) \mathrm{Se}$ and $128.3 \mathrm{mg}(4 \mathrm{mmol}) \mathrm{S}$, dissolved together in $3 \mathrm{~mL}$ of trioctylphosphine (TOP) were rapidly injected into the flask followed by a temperature reduction to $300^{\circ} \mathrm{C}$. After $10 \mathrm{~min}$, the mixture was cooled to room temperature and the QDs were purified two times by washing them with $20 \mathrm{~mL}$ chloroform $\left(\mathrm{CHCl}_{3}\right)$ and an excess of acetone. These QDs showed photoluminescence quantum yields (PL-QYs) between $40 \%$ and $80 \%$. Finally, the QDs were redispersed in $4 \mathrm{~mL}$ of $\mathrm{CHCl}_{3}$ and either used directly or after a subsequent phase transfer for crystallization. The $\operatorname{lnZnP}$ cores were synthesized by mixing $35.4 \mathrm{mg}(0.12 \mathrm{mmol}) \ln (\mathrm{OAc})_{3}$, $13.8 \mathrm{mg}(0.06 \mathrm{mmol}) \mathrm{Zn}(\mathrm{OAc})_{2} \cdot 2 \mathrm{H}_{2} \mathrm{O}, 94.2 \mathrm{mg}(0.36 \mathrm{mmol})$ palmitic acid, and $8 \mathrm{~mL} O D E$ in a $25 \mathrm{~mL}$ three-necked flask. The suspension was degassed twice, heated to $110^{\circ} \mathrm{C}$, and degassed again for $30 \mathrm{~min}$. After backfilling with Ar and heating to $300^{\circ} \mathrm{C}, 17.4 \mu \mathrm{L}(0.06 \mathrm{mmol})(\mathrm{TMS})_{3} \mathrm{P}$ in $1 \mathrm{~mL}$ ODE were rapidly injected into the flask and held at $230{ }^{\circ} \mathrm{C}$ for $2 \mathrm{~h}$. For the $\mathrm{GaP}$ shell, a solution of $5 \mathrm{mg}(0.03 \mathrm{mmol}) \mathrm{GaCl}_{3}$ and $31.5 \mu \mathrm{L}$ $(0.1 \mathrm{mmol}$ ) oleic acid in $2 \mathrm{~mL}$ ODE was added dropwise to the $\operatorname{lnZnP}$ core solution at $200{ }^{\circ} \mathrm{C}$. For the $\mathrm{ZnS}$ shell formation, $55 \mathrm{mg}(0.3 \mathrm{mmol})$ $\mathrm{Zn}(\mathrm{OAc})_{2}$ were added to the reaction flask at room temperature and the suspension was heated to $230{ }^{\circ} \mathrm{C}$ for $4 \mathrm{~h}$. Finally, $117 \mu \mathrm{L}(0.5 \mathrm{mmol})$ 1-dodecanethiol were slowly injected into the flask and the mixture was kept at $230{ }^{\circ} \mathrm{C}$ for further $2 \mathrm{~h}$. The obtained multishell QDs were purified three times by precipitation with acetone/methanol $(\mathrm{MeOH})$ and redispersed in toluene, exhibiting a PL-QY of $\approx 70 \%$. The CdSe/ZnS and $\operatorname{InZnP} / \mathrm{GaP} / \mathrm{ZnS}$ QDs were phase-transferred into water by diluting $30 \mu \mathrm{L}$ of the nanocrystals with $500 \mu \mathrm{L}$ of $\mathrm{CHCl}_{3}$ and stirred with $1 \mathrm{~mL}$ of $0.2 \mathrm{M} \mathrm{MPA}$ at $\mathrm{pH} 10$ for $2 \mathrm{~h}$. The phases were separated, and the aqueous phase was used without further purification. ${ }^{[43]}$

Preparation of Mixed Crystals: The fast preparation of the mixed crystals using the newly developed LLDC-technique with aqueous suspension of CdTe QDs was performed by firstly placing $5 \mathrm{~mL}$ of $\mathrm{MeOH}$ in a glass vial. Subsequently, a mixture of $0.5 \mathrm{~g} \mathrm{NaCl}$ and $1.7 \mathrm{~mL}$ CdTe QDs was slowly injected to the bottom of the vial, forming an aqueous layer below the $\mathrm{MeOH}$ with a stable interface between the two liquids. Due to the spontaneous diffusion of $\mathrm{MeOH}$ into the $\mathrm{NaCl}$ solution, the solubility of the salt is gradually decreased which leads to the formation of mixed crystals within 15-20 h. For the phase transferred $\mathrm{CdSe} / \mathrm{ZnS}$ and $\operatorname{lnZnP} / \mathrm{GaP} / \mathrm{ZnS}$ QDs, a similar approach is used. Preparing mixed crystals directly from organic solvent-based QDs uses a modified, two-step LLDC-approach. Here, first $3 \mathrm{~mL}$ of saturated $\mathrm{NaCl}$ in $\mathrm{MeOH}$ are injected into $2 \mathrm{~mL}$ of a diluted QD solution $(50 \mu \mathrm{L}$ QDs and $1950 \mu \mathrm{L} \mathrm{CHCl}{ }_{3}$ ). Within this mixture, seed $\mathrm{NaCl}$ crystals are formed within seconds, causing a slight turbidity of the solution. The solution is then centrifuged for $3 \mathrm{~min}$ at $3260 \mathrm{~g}$ to separate the supernatant solution from the seeds, which are washed three times with $\mathrm{MeOH}$ and redispersed in $5 \mathrm{~mL}$ of pure $\mathrm{MeOH}$ afterwards. To form the proper mixed crystals, $1.7 \mathrm{~mL}$ of water dissolving $0.5 \mathrm{~g} \mathrm{NaCl}$ is injected under the seed-MeOH layer and stored for $20 \mathrm{~h}$. A picture of the seed- $\mathrm{MeOH}-$ water mixture for different CdSe/ZnS QDs can be found in Figure S1, Supporting Information.

Stability Test: The prepared mixed crystals, either with aqueous or organic-based QDs, were placed in a $0.5 \mathrm{M}$ solution of benzoyl peroxide, a strong oxidizing agent, in toluene for $24 \mathrm{~h}$ to confirm that the QDs are incorporated into the salt matrix. The same test was performed with the pure QDs (for the $\mathrm{CHCl}_{3}$ soluble QDs) and, as a comparison, with mixed crystals prepared by our classical method. ${ }^{[31]}$

LED Preparation: The mixed crystals were milled to a fine powder, mixed with a two-component silicone resin (ACC Silicones), and blended on a blue-emitting commercial light emitting diode (LED). The silicone was hardened at $60^{\circ} \mathrm{C}$ for $2 \mathrm{~h}$, encapsulating the mixed crystals powder on top of the LED-chip.

Preparation of Thin Sheets for Transmission Electron Microscopy (TEM): The used epoxy resin Araldite 6005 Kit (Electron Microscopy Sciences) was warmed and the components were mixed according to ref. [44] filled into the mold, and the mixed crystals were placed within the resin. After curing at $60{ }^{\circ} \mathrm{C}$, the epoxy-mixed crystal composite was trimmed and sectioned into $200 \mathrm{~nm}$ thin sheets, using an ultramicrotome and an ethanol bath. The sheets were placed on 200 mesh carbon-coated copper grids for the subsequent TEM investigations.

Characterization: Photoluminescence (PL) measurements were performed at room temperature using a FluoroMax-4 spectrofluorometer (Horiba Jobin Yvon). PL-lifetime (PL-LT) measurements were performed using a FluoroLog-3 spectrofluorometer (Horiba Jobin Yvon) equipped with a pulsed LED diode and a TCSPC module at room temperature. Average PL-lifetimes were calculated at the time, where the initial signal intensity was reduced to 10 000/e. Absolute PL-quantum yield (PL-QY) measurements were performed using a FluoroLog-3 spectrofluorometer (Horiba Jobin Yvon) equipped with a Quanta- $\phi$ integrating sphere. UVvis absorption spectra were recorded using a Cary 50 spectrophotometer (Varian). PL-QY measurements of colloidal QD solutions were performed as described in ref. [45] by a comparison with Rhodamine 6C and Rhodamine 101 (both Radiant Dyes Laser) in ethanol (Uvasol, Merck), assuming their PL-QY as $91.0 \%$ and $91.5 \%$, respectively. Microscopic investigations of the mixed crystals were performed on a Zeiss Axiostar plus fluorescence microscope on glass slides. TEM measurements were performed using a FEI Tecnai G2 F30. Thin sheets for the TEM measurements were prepared following the method of Glauert using a Leica ultramicrotome.

\section{Supporting Information}

Supporting Information is available from the Wiley Online Library or from the author.

\section{Acknowledgements}

M.A. and Z.W. contributed equally to this work. This work was financially supported by the EU-FP7 Network of Excellence Nanophotonics for Energy Efficiency (N4E) as well as by the BMBF TUR 09/001 and TUBITAK 112E183. Z.W. greatly acknowledges a fellowship provided by the Alexander von Humboldt Foundation. A.D. acknowledges the European Social Fund (ESF) within the project "ChemIT" and the Cluster of Excellence "Center for Advancing Electronics Dresden." Z.S.-E. and H.V.D. also acknowledge the financial support from TUBITAK EEEAG 109E002, 109E004, 110E010, 110E217, and from NRF-RF-2009-09, NRF-CRP-6-2010-02, and A*STAR of Singapore. H.V.D. also thanks for generous support from ESF-EURYI and TUBA-GEBIP.

Received: February 9, 2015

Revised: February 27, 2015

Published online: March 24, 2015

[1] A. Henglein, Ber. Bunsenges. Phys. Chem. 1982, 86, 301.

[2] R. Rossetti, S. Nakahara, L. E. Brus, J. Chem. Phys. 1983, 79, 1086.

[3] H. Weller, U. Koch, M. Gutiérrez, A. Henglein, Ber. Bunsenges. Phys. Chem. 1984, 88, 649.

[4] M. L. Steigerwald, A. P. Alivisatos, J. M. Gibson, T. D. Harris, R. Kortan, A. J. Muller, A. M. Thayer, T. M. Duncan, D. C. Douglass, L. E. Brus, J. Am. Chem. Soc. 1988, 110, 3046.

[5] T. Rajh, O. I. Micic, A. J. Nozik, J. Phys. Chem. 1993, 97, 11999.

[6] V. L. Colvin, M. C. Schlamp, A. P. Alivisatos, Nature 1994, 370, 354.

[7] V. I. Klimov, A. A. Mikhailovsky, S. Xu, A. Malko, J. A. Hollingsworth, C. A. Leatherdale, H.-L. Eisler, M. G. Bawendi, Science 2000, 290, 314.

[8] V. I. Klimov, S. A. Ivanov, J. Nanda, M. Achermann, I. Bezel, J. A. McGuire, A. Piryatinski, Nature 2007, 447, 441. 
[9] H. V. Demir, S. Nizamoglu, T. Erdem, E. Mutlugun, N. Gaponik, A. Eychmüller, Nano Today 2011, 6, 632.

[10] B. Guzelturk, Y. Kelestemur, M. Olutas, S. Delikanli, H. V. Demir ACS Nano 2014, 8, 6599.

[11] R. Vogel, P. Hoyer, H. Weller, J. Phys. Chem. 1994, 98, 3183.

[12] A. L. Rogach, A. Eychmüller, S. G. Hickey, S. V Kershaw, Small 2007, 3,536 .

[13] D. V Talapin, J.-S. Lee, M. V Kovalenko, E. V Shevchenko, Chem Rev. 2010, 110, 389

[14] J. Poppe, S. G. Hickey, A. Eychmüller, J. Phys. Chem. C 2014, 118, 17123.

[15] C. B. Murray, D. J. Norris, M. G. Bawendi, J. Am. Chem. Soc. 1993 $115,8706$.

[16] N. Gaponik, D. V Talapin, A. L. Rogach, K. Hoppe, E. V Shevchenko, A. Kornowski, A. Eychmüller, H. Weller, J. Phys. Chem. B 2002, 106, 7177.

[17] D. V. Talapin, A. L. Rogach, A. Kornowski, M. Haase, H. Weller, Nano Lett. 2001, 1, 207.

[18] A. B. Greytak, P. M. Allen, W. Liu, J. Zhao, E. R. Young, Z. Popović, B. J. Walker, D. G. Nocera, M. G. Bawendi, Chem. Sci. 2012, 3, 2028.

[19] L. Bakueva, S. Musikhin, M. A. Hines, T.-W. F. Chang, M. Tzolov, G. D. Scholes, E. H. Sargent, Appl. Phys. Lett. 2003, 82, 2895.

[20] P. O. Anikeeva, J. E. Halpert, M. G. Bawendi, V. Bulović, Nano Lett. 2009, 9, 2532

[21] N. Gaponik, S. G. Hickey, D. Dorfs, A. L. Rogach, A. Eychmüller, Small 2010, 6, 1364.

[22] S. K. Panda, S. G. Hickey, H. V. Demir, A. Eychmüller, Angew. Chem. Int. Ed. 2011, 50, 4432

[23] X. Zhang, Y. Zhang, Y. Wang, S. Kalytchuk, S. V Kershaw, Y. Wang, P. Wang, T. Zhang, Y. Zhao, H. Zhang, T. Cui, Y. Wang, J. Zhao, W. W. Yu, A. L. Rogach, ACS Nano 2013, 7, 11234.

[24] W. Zhang, Q. Lou, W. Ji, J. Zhao, X. Zhong, Chem. Mater. 2014, 26, 1204.

[25] E. Jang, S. Jun, H. Jang, J. Lim, B. Kim, Y. Kim, Adv. Mater. 2010, 22, 3076.

[26] QD Vision.
[27] H. Zhang, Z. Cui, Y. Wang, K. Zhang, X. Ji, C. Lü, B. Yang, M. Gao, Adv. Mater. 2003, 15, 777.

[28] J. Czochralski, Z. Phys. Chem. 1917, 92, 219.

[29] Y. Chen, J. Vela, H. Htoon, J. L. Casson, D. J. Werder, D. A. Bussian, V. I. Klimov, J. A. Hollingsworth, J. Am. Chem. Soc. 2008, 130, 5026.

[30] Z. Popović, W. Liu, V. P. Chauhan, J. Lee, C. Wong, A. B. Greytak, N. Insin, D. G. Nocera, D. Fukumura, R. K. Jain, M. G. Bawendi, Angew. Chem. 2010, 122, 8831.

[31] T. Otto, M. Müller, P. Mundra, V. Lesnyak, H. V. Demir, N. Gaponik, A. Eychmüller, Nano Lett. 2012, 12, 5348.

[32] M. Müller, M. Kaiser, G. M. Stachowski, U. Resch-Genger, N. Gaponik, A. Eychmüller, Chem. Mater. 2014, 26, 3231.

[33] S. Kalytchuk, O. Zhovtiuk, A. L. Rogach, Appl. Phys. Lett. 2013, 103, 103105.

[34] T. Erdem, Z. Soran-Erdem, P. L. Hernandez-Martinez, V. K. Sharma, H. Akcali, I. Akcali, N. Gaponik, A. Eychmüller, H. V. Demir, Nano Res. 2014, 8, 860

[35] M. Adam, R. Tietze, N. Gaponik, A. Eychmüller, Z. Phys. Chem. 2014, 229, 109.

[36] J. Burgees, Metal Ions in Solution, Ellis Horwood, Hemel Hempsted, UK 1981, p 481.

[37] H. J. Meyer, Meyers Großes Konversations-Lexikon, Bibliographisches Institut, Leipzig, Wien 1909.

[38] W. K. Bae, K. Char, H. Hur, S. Lee, Chem. Mater. 2008, 20, 531.

[39] M. Grabolle, J. Ziegler, A. Merkulov, T. Nann, U. Resch-Genger, Ann. N.Y. Acad. Sci. 2008, 1130, 235.

[40] A. Shavel, N. Gaponik, A. Eychmüller, J. Phys. Chem. B 2006, 110, 19280

[41] W. K. Bae, J. Kwak, J. Lim, D. Lee, M. K. Nam, K. Char, C. Lee, S. Lee, Nano Lett. 2010, 10, 2368.

[42] S. Kim, T. Kim, M. Kang, S. K. Kwak, T. W. Yoo, L. S. Park, I. Yang, S. Hwang, J. E. Lee, S. K. Kim, S.-W. Kim, J. Am. Chem. Soc. 2012, 134,3804

[43] S. Tamang, G. Beaune, I. Texier, P. Reiss, ACS Nano 2011, 5, 9392.

[44] A. M. Glauert, Microsc. Anal. 1991, 25, 15.

[45] C. Würth, M. Grabolle, J. Pauli, M. Spieles, U. Resch-Genger, Nat. Protoc. 2013, 8, 1535 